\title{
A NOTE ON SUBPARACOMPACT SPACES
}

\author{
R. E. HODEL
}

ABstract. The main result in this paper states that every metacompact space in which every closed set is a $G_{\delta}$ is subparacompact. The class of countably subparacompact spaces is introduced and several results about such spaces are proved.

1. Introduction. A topological space is said to be subparacompact if every open cover has a $\sigma$-discrete closed refinement. This class of spaces was introduced by McAuley in [8] (he called such spaces $F_{\sigma}$-screenable) and more recently has been studied by Burke [2] and Creede [3]. Every paracompact space is subparacompact, and Creede proved that every semistratifiable space is subparacompact. The main result of this paper is the following theorem.

Theorem 1. Every metacompact space in which every closed set is a $G_{\delta}$ is subparacompact.

In connection with this result it should be pointed out that Worrell [9] has given an example of a metacompact space which fails to be subparacompact.

In $\$ 2$ we give the proof of Theorem 1 . In $\$ 3$ we introduce the class of countably subparacompact spaces and prove several results about such spaces. Finally, in $\$ 4$ we summarize the theorems in this paper together with some results from the literature.

A topological space is metacompact if every open cover has a point finite open refinement. According to Bing [1] a topological space is screenable if every open cover has a $\sigma$-disjoint open refinement. No separation axioms are assumed in this paper.

2. Proof of Theorem 1. The following notation will be used in this section: given a set $X$, a point $p$ in $X$, and a cover $\mathcal{u}$ of $X$, ord $(p, \mathcal{u})$ is the number of elements of $u$ containing $p$. Theorem 1 follows immediately from this Lemma.

Lemma. Let $X$ be a topological space in which every closed set is a $G_{\delta}$. Then every point finite open cover of $X$ has a $\sigma$-discrete closed refinement.

Proof. Let $\mathcal{u}=\left\{U_{\alpha}: \alpha\right.$ in $\left.A\right\}$ be a point finite open cover of $X$,

Received by the editors August 12, 1969.

AMS Subject Classifications. Primary 5420; Secondary 5440.

Key Words and Phrases. Metacompact, G - $_{-}$set, subparacompact, screenable, countably subparacompact, countably metacompact. 
let $\leqq$ be a well ordering for $A$. We shall construct a $\sigma$-discrete closed refinement $\mathcal{F}$ of $\mathcal{u}$. For $n=1,2, \cdots$ let $F_{n}=\{x$ in $X: \operatorname{ord}(x, \mathfrak{u}) \leqq n\}$. It is easy to check that $F_{n}$ is a closed set and so $F_{n}=\bigcap_{i=1}^{\infty} W_{n i}$, where each $W_{n i}$ is an open set. Since each $U_{\alpha}$ is an open set we have $U_{\alpha}=\bigcup_{j=1}^{\infty} K_{\alpha j}$, where each $K_{\alpha j}$ is a closed set. For $n=1,2, \cdots$, $i=1,2, \cdots, j=1,2, \cdots$, and $\alpha$ in $A$ let

$$
H_{\alpha n i j}=\left(K_{\alpha j}-\bigcup_{\beta<\alpha} U_{\beta}\right) \cap\left(F_{n}-W_{(n-1) i}\right) \quad\left(W_{0 i}=\varnothing\right),
$$

let $\mathfrak{F}_{n i j}=\left\{H_{\alpha n i j}: \alpha\right.$ in $\left.A\right\}$, and let $\mathfrak{H}=\bigcup_{n=1}^{\infty} \bigcup_{i=1}^{\infty} \bigcup_{j=1}^{\infty} \mathfrak{F}_{n i j}$.

Clearly each element of $\mathfrak{T}$ is closed and is contained in some element of $\mathcal{u}$. To see that $\mathcal{H}$ covers $X$, consider $p$ in $X$. Let $\alpha$ be the smallest element of $A$ such that $p$ belongs to $U_{\alpha}$, and let $\operatorname{ord}(p, \mathfrak{u})=n$. Then there is some $i$ such that $p$ is not in $W_{(n-1) i}$ and there is some $j$ such that $p$ is in $K_{\alpha j}$. Thus $p$ is in $H_{\alpha n i j}$.

It remains to show that each $\mathscr{H}_{n i j}$ is a discrete collection. Let $p$ be an arbitrary point of $X$. If $\operatorname{ord}(p, \mathfrak{u})>n$ then $p$ has a neighborhood which misses $F_{n}$ and so $p$ has a neighborhood which misses all elements of $\mathfrak{F}_{n i j}$. If $\operatorname{ord}(p, \mathcal{u})<n$ then $W_{(n-1) i}$ is a neighborhood of $p$ which misses all elements of $\mathfrak{F}_{n i j}$. Finally suppose ord $(p, \mathcal{U})=n$ with $p$ belonging to $U_{\alpha_{1}}, \cdots, U_{\alpha_{n}}, \alpha_{1}<\cdots<\alpha_{n}$, let $V=\bigcap_{i-1}^{n} U_{\alpha_{i}}$, and consider $\alpha$ in $A$. If $\alpha<\alpha_{1}$ then $V \cap H_{\alpha n i j}=\varnothing$ since $x$ in $V \cap H_{\alpha n i j}$ implies $\operatorname{ord}(x, \mathcal{u})>n$ and $x$ in $F_{n}$, a contradiction. If $\alpha>\alpha_{1}$ then $U_{\alpha_{1}} \cap H_{\alpha n i j}=\varnothing$ and so $V \cap H_{\alpha n i j}=\varnothing$. Thus $V$ is a neighborhood of $p$ which intersects at most one element of $\mathfrak{H}_{n i j}$, namely $H_{\alpha_{1} i j}$.

3. Countably subparacompact spaces. A topological space is said to be countably subparacompact if every countable open cover has a $\sigma$-discrete closed refinement. This concept was studied by Mansfield in [7], where he proved that in normal spaces countable subparacompactness is equivalent to countable paracompactness.

It is easy to show that a topological space $X$ is countably subparacompact if and only if it satisfies this condition: given a countable open cover $\left\{U_{n}: n=1,2, \cdots\right\}$ of $X$, there is a countable closed cover $\left\{F_{n j}: n=1,2, \cdots, j=1,2, \cdots\right\}$ of $X$ with $F_{n j} \subseteq U_{n}$ for all $n$ and $j$. Theorem 2 below follows immediately from this characterization of countable subparacompactness.

THEOREM 2. Every topological space in which every closed set is a $G_{\delta}$ is countably subparacompact.

The relation between countable metacompactness and countable subparacompactness is as follows. 
TheOREM 3. Every countably subparacompact space is countably metacompact.

Proof. Let $\left\{U_{n}: n=1,2, \cdots\right\}$ be a countable open cover of $X$. It suffices to construct a point finite open cover $\left\{V_{n}: n=1,2 \cdots\right\}$ of $X$ with $V_{n} \subseteq U_{n}$ for all $n$. Let $\left\{F_{n j}: n=1,2, \cdots, j=1,2, \cdots\right\}$ be a closed cover of $X$ with $F_{n j} \subseteq U_{n}$ for all $n$ and $j$. Let $V_{1}=U_{1}$ and for $n=2,3, \cdots$ let $V_{n}=U_{n}-U\left\{F_{k j}: k<n, j<n\right\}$. It is easy to check that $\left\{V_{n}: n=1,2, \cdots\right\}$ is the desired point finite open cover of $X$.

EXample. A countably metacompact space not countably subparacompact. In [2] Burke gives an example of a metacompact space $X$ which fails to be subparacompact. It is easy to check that the space $X$ is also screenable. It follows from Theorem 4 below that $X$ cannot be countably subparacompact.

Theorem 4. Every screenable countably subparacompact space is subparacompacl.

Proof. Let $W$ be an open cover of $X$. We want to construct a $\sigma$-discrete closed refinement $\mathcal{H C}$ of $\mathcal{W}$. Let $\bigcup_{n=1}^{\infty} \mathcal{U}_{n}$ be a $\sigma$-disjoint open refinement of $\mathscr{W}$, and for $n=1,2, \cdots$ let $U_{n}=U \mathcal{U}_{n}$. Then $\left\{U_{n}: n\right.$ $=1,2, \ldots\}$ is a countable open cover of $X$ so there is a closed cover $\left\{F_{n j}: n=1,2, \cdots, j=1,2, \cdots\right\}$ of $X$ with $F_{n j} \subseteq U_{n}$ for all $n$ and $j$. Let $\mathcal{F}_{n j}=\left\{U \cap F_{n j}: U \in \mathcal{U}_{n}\right\}$ and let $\mathcal{H C}=\bigcup_{n=1}^{\infty} \bigcup_{j=1}^{\infty} \mathcal{F C}_{n j}$. Clearly $\mathfrak{H C}$ covers $X$ and each element of $\mathfrak{H C}$ is contained in some element of $W$. Each element of $\mathcal{H C}$ is closed since $F_{n j} \cap U=F_{n j}$ $\cap\left(X-\cup\left\{U^{\prime}: U^{\prime} \in \mathcal{u}_{n}, U^{\prime} \neq U\right\}\right)$, and it is easy to verify that each $\mathfrak{F}_{n j}$ is discrete. Thus $\mathfrak{H}$ is the desired $\sigma$-discrete closed refinement of $w$.

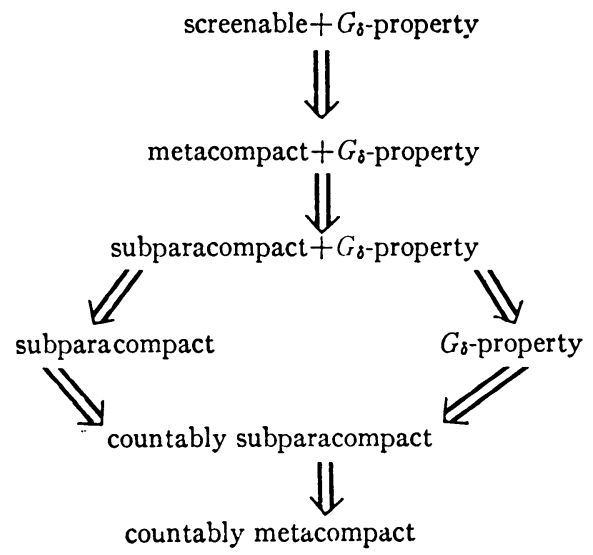


4. Summary. For convenience we shall say that a topological space has the $G_{\delta}$-property if every closed set is a $G_{\delta}$. Heath [6] proved that every screenable space with the $G_{\delta}$-property is metacompact, and Greever [4], [5] generalized this result by showing that every space with the $G_{\delta}$-property is countably metacompact and that every screenable countably metacompact space is metacompact. These results, together with the theorems in this paper, can be summarized in the preceding diagram.

\section{REFERENCES}

1. R. H. Bing, Metrization of topological spaces, Canad. J. Math. 3 (1951), 175186. MR 13, 264.

2. D. K. Burke, On subparacompact spaces, Proc. Amer. Math. Soc. 23 (1969), 655-663.

3. G. Creede, Semi-stratifiable spaces, Topology Conference Arizona State University, Tempe, Ariz., 1967, pp. 318-323.

4. J. Greever, On spaces in which every closed set is a $G_{\delta}$, Proc. Japan Acad. 43 (1967), 445-447. MR 37 \#2183.

5. —_, On screenable topological spaces, Proc. Japan Acad. 44 (1968), 434-438. MR $37 \# 3520$.

6. R. W. Heath, Screenability, pointwise paracompactness, and metrization of Moore spaces, Canad. J. Math. 16 (1964), 763-770. MR 29 \#4033.

7. M. J. Mansfield, On countably paracompact normal spaces, Canad. J. Math. 9 (1957), 443-449. MR 19, 436.

8. L. F. McAuley, A note on complete collectionwise normality and paracompactness, Proc. Amer. Math. Soc. 9 (1958), 796-799. MR 20 \#6086.

9. J. M. Worrell, Jr., The closed continuous images of metacompact topological spaces, Portugal. Math. 25 (1966), 175-179. MR 38 \#676.

Duke University, Durham, North Carolina 27706 